\title{
Back to Bedside!
}

\section{Hans Stalder}

Prof. Dr méd., membre de la rédaction

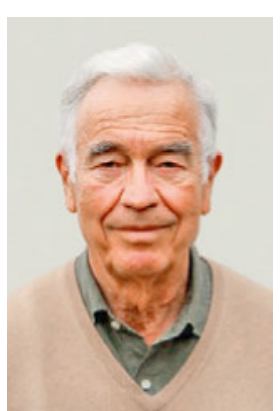

On aurait pu penser que l'introduction du dossier électronique ferait gagner du temps aux internes de nos hôpitaux pour s'occuper davantage des patients. Or, on observe le contraire. Selon une étude du Service de Médecine interne générale du CHUV, les internes s'occupent de leurs patients seulement 1,7 heure par jour et restent 3 fois plus longtemps derrière un ordinateur [1]. Ce résultat est conforme à d'autres études effectuées dans d'autres centres universitaires.

Cela pose des questions concernant aussi bien l'adéquation des soins aux malades que le bien-être des médecins menacés de burnout et il est rassurant de constater que les responsables du service mentionné entreprennent des actions pour changer cet état de choses.

II y a cependant un point tout aussi inquiétant et guère mentionné dans ces études: l'effet préjudiciable du travail administratif sur la formation postgraduée. $\mathrm{Au}$ CHUV, les internes travaillant le jour reçoivent que 35 minutes de formation, dont seulement une partie est structurée, et l'équipe du soir que 3 minutes! Cela contraste avec la description du programme de formation de leur clinique, qui déclare $71 / 2$ heures hebdomadaires de formation théorique (cours, journal club, etc.) et 3 heures de formation pratique obligatoires et prévoit 4-6 heures d'enseignement au lit du malade.

On peut donc se demander comment les exigences de base de la formation postgraduée requises par l'Institut Suisse de Formation en Médecine (ISFM [2]) peuvent être respectées, comme - pour n'en citer que quelquesunes - "procéder à une anamnèse concise, pratiquer un examen clinique, interpréter les informations obtenues lors de l'anamnèse et de l'examen clinique, bâtir des relations de confiance avec les patients, leurs familles et les autres proches, communiquer de manière compréhensible pour la personne les risques et les avantages des mesures diagnostiques et thérapeutiques et obtenir un consentement éclairé», etc., sans parler des centaines d'objectifs spécifiques définis par la Société de Médecine interne générale. Par ailleurs, l'ISFM ajoute que "seuls peuvent être reconnus les établissements de for- mation où le contact physique avec les patients est garanti». On peut se demander si 1,7 heure par jour remplit cette exigence. Petite consolation: on peut espérer que l'objectif «Le spécialiste documente les résultats de l'anamnèse, du status et des examens complémentaires ainsi que le plan de traitement de manière compréhensible et pertinente» sera atteint!

Il est certainement louable que l'ISFM et les sociétés de spécialités aient défini les objectifs généraux de formation. Mon expérience en tant qu'expert lors des visites d'accréditation m'a appris qu'ils ne sont pratiquement jamais atteints et cela particulièrement dans les services hospitaliers. Pression économique, DRGs, diminution de la durée des hospitalisations, qui servent de plus en plus à des interventions techniques ponctuelles, horaire de travail de 50 heures, dont une part croissante est consacrée au travail administratif, ne contribuent guère à une ambiance de formation postgraduée adéquate, en particulier pour les futurs médecins de famille.

"Back to Bedside» [3] est l'objectif d'une enquête faite par 37 jeunes médecins pour trouver et inventer les meilleurs milieux pour apprendre la médecine et pour augmenter le sens du travail. Peut-on remodeler le travail des internes en milieu hospitalier afin qu'il soit adapté à la formation en médecine de famille? Ou faudrait-il déplacer ailleurs les moyens importants que la société investit pour la formation? Si on veut vraiment assurer une relève adéquate des médecins de premier recours [4], il faut repenser leur formation de fond en comble!

\section{Références}

1 Wenger N, Méan M, Castioni J, Marques-Vidal P, Waeber G, Garnier A. Allocation of Internal Medicine Resident Time in a Swiss Hospital: A Time and Motion Study of Day and Evening Shifts. Ann Intern Med. 2017;166:579-86.

2 Institut Suisse de formation médicale. https://www.fmh.ch/ files/pdf17/allg_lz_f.pdf.

3 Hipp DM, Rialon KL, Nevel K, Kothari AN, Jardine LDA. Back to Bedside: Residents' and Fellows' Perspectives on Finding Meaning in Work. J Grad Med Educ. 2017;9:269-73.

4 Gaspoz JM, Héritier F, Aujesky D, Capaul R, Providoli R, Tronnolone D, Zogg F, Häfliger Berger B. Médecine Interne Générale: la relève au centre des préoccupations. Bull Méd Suisses. 2018;99(10):300-2. 